\title{
Die Zukunft der Bushaltestelle vor dem Hintergrund von Mobility-as-a-Service - Eine qualitative Betrachtung des öffentlichen Personennahverkehrs in Deutschland
}

\author{
Andreas Hahn • Christina Pakusch (iD) $\cdot$ Gunnar Stevens
}

Eingegangen: 2. August 2019 / Angenommen: 22. Januar 2020 / Online publiziert: 4. Februar 2020

(C) Der/die Autor(en) 2020

Zusammenfassung Die Bundesrepublik Deutschland erlebt in jüngster Vergangenheit verstärkt Dieselfahrverbote in Großstädten. Gleichzeitig erfahren Großstädte als Lebensmittelpunkt eine steigende Beliebtheit. Für Verkehrsunternehmen gilt es, der Bevölkerung nachhaltige Mobilitätslösungen zu bieten, die ein Höchstmaß an Flexibilität ermöglichen. Moderne Mobility-as-a-Service-Konzepte und Innovationen in der Mobilität stellen den klassischen, planorientierten, öffentlichen Personennahverkehr und damit auch die Existenz von Bushaltestellen infrage. Mittels qualitativer Experten-Interviews lässt sich feststellen, dass sich die Bushaltestelle in den Innenstädten vor dem Hintergrund zunehmender digitaler Vernetzung von Mobilitätsanbietern und daraus resultierender modernen Mobility-as-a-service-Konzepte verändern wird. Die Ergebnisse deuten darauf hin, dass die Bushaltestelle in den Innenstädten auch in Zukunft bestehen bleibt und um ,on demand“-Verkehre ergänzt wird. Ein radikaler Wandel, wie eine flächendeckende Einführung von autonom fahrenden Bussen, könnte langfristig eine Runderneuerung der Haltestelle zur Folge haben.

Schlüsselwörter Innerstädtische Bushaltestelle · Mobilität · Öffentlicher Personennahverkehr · Qualitative Interviews · Digitalisierung · Dynamische Fahrgastinformationen

Zusatzmaterial online Zusätzliche Informationen sind in der Online-Version dieses Artikels (https:// doi.org/10.1365/s40702-020-00589-9) enthalten.

\footnotetext{
A. Hahn $\cdot$ C. Pakusch $(\bowtie) \cdot$ G. Stevens

Fachbereich Wirtschaftswissenschaften, Campus Sankt Augustin, Hochschule Bonn-Rhein-Sieg, Grantham-Allee 20, 53757 Sankt Augustin, Deutschland

E-Mail: christina.pakusch@h-brs.de
} 


\title{
The Future of the Bus Stop Against the Background of Mobility as a Service-A Qualitative View of Local Public Transport in Germany
}

\begin{abstract}
In the recent past, the Federal Republic of Germany has experienced an increase in diesel driving bans in large cities. At the same time, large cities are becoming increasingly popular as the centre of life. Transport companies need to offer the population sustainable mobility solutions that enable maximum flexibility. Modern Mobility-as-a-Service-concepts and innovations in mobility are questioning the classic, plan-oriented, local public transport system and thus also the existence of bus stops. Qualitative expert interviews show that bus stops in city centres will change: One reason being the increasing digital networking of mobility providers and the resulting modern Mobility-as-a-Service-concepts. The results indicate that the bus stop in the inner cities will remain in the future and will be supplemented by on-demand traffic. A radical change, such as the nationwide adoption of autonomous buses, could lead to a complete renewal of the bus stop in the long term.
\end{abstract}

Keywords Urban bus stop · Mobility · Public transport - Qualitative interviews · Digitalisation $\cdot$ Dynamic passenger information

\section{Einleitung}

Im Zusammenhang mit dem zunehmenden Wunsch der Menschen in Großstädten zu wohnen und dem daraus resultierenden stetigen Wachstum der Innenstädte lässt sich ein erhöhter Bedarf an möglichst individuellen und flexiblen Mobilitätslösungen erkennen (Moeckel und Nagel 2016). Die Folgen dieser veränderten Bedarfe machen eine Veränderung hin zu modernen und ganzheitlichen Mobilitätskonzepten erforderlich, um die flächenmäßig eng besiedelten Großstädte optimal mit Mobilität zu versorgen. Politik, Städte- und Verkehrsplaner sowie Verkehrsbetriebe stehen also vor der Herausforderung, diese Konzepte möglichst zukunftsorientiert, nachhaltig und dennoch ökonomisch effizient zu gestalten. Den Verkehrsunternehmen kommt dabei die Aufgabe zu, mit ihren Bus- und Bahnverkehren einen Großteil der öffentlichen kollektiven Personenmassenverkehre zu bewältigen. Neben den herkömmlichen motorisierten Individualverkehren wird auch der öffentliche Personennahverkehr (ÖPNV) zunehmend digitalisiert. Durch die digitale Vernetzung von Mobilitätsanbieter und -konsument werden Mobility-as-a-Service (MaaS)-Konzepte ermöglicht (Kamargianni et al. 2016; Arsenio et al. 2016).

Diese MaaS-Konzepte kombinieren öffentliche und private Verkehrsangebote mittels einheitlichem digitalen Zugangsportal. Integraler Bestandteil solcher MaaS-Konzepte sind individualisierte, dynamische und flexible Mobilitätslösungen, die sich an den konkreten Bedarfen der Nutzer orientieren und ihnen für jeden Weg eine maßgeschneiderte Mobilitätslösung anbieten. Diese Konzepte sowie Innovationen in der Mobilität, wie vollautomatisiertes Fahren, stellen den klassischen planorientierten ÖPNV und damit auch die Existenz von Bushaltestellen infrage.

Die Haltestelle fungiert als der Ort, der Fahrgästen das $\mathrm{Zu}$ - oder Aussteigen in öffentliche Verkehrsmittel ermöglicht und dient Nahverkehrsunternehmen somit 
als Sammelstelle der Kunden für den kollektiven ÖPNV. Für den Kunden bilden Haltestellen ein erkennbares Gesicht der Nahverkehrsunternehmen. Derzeit haben Fahrgäste nur dann die Möglichkeit die Verkehrsangebote wahrzunehmen, wenn sie eine Haltestelle nutzen. Neben dem räumlichen Zusammenführen von Mobilitätsangebot und -nachfrage dienen Haltestellen hauptsächlich der Information der Fahrgäste über Fahrpläne, Liniennetze, Tarife, Tickets und Beeinträchtigungen des Betriebs. Manche Haltestellen werden zunehmend als Marketinginstrument genutzt.

Im Rahmen dieses wissenschaftlichen Papiers untersuchen wir die Bedeutung und mögliche Veränderung der Bushaltestelle in deutschen Innenstädten vor dem Hintergrund zunehmend flexibilisierter Mobilitätskonzepte. Heute ist eine Beförderung ohne feste Haltestelle nicht möglich. Aufgrund der fortschreitenden Digitalisierung der Mobilität kann sich dieser Status quo jedoch rasant verändern bis hin zur kompletten Abschaffung der fixen Haltestelle. Die konkrete Forschungsfrage lautet: „Welche Veränderungen erfährt die Bushaltestelle in den deutschen Großstädten unter Berücksichtigung von Mobility-as-a-Service-Konzepten?“

Das Paper ist wie folgt aufgebaut: Zunächst präsentieren wir die Ergebnisse der Literaturrecherche, bevor wir die Methodik der qualitativen Interviews darstellen. Anschließend stellen wir die Ergebnisse der acht Experteninterviews dar, mit denen wir die Bedeutung der Bushaltestelle in den deutschen Innenstädten und deren zukünftige Entwicklung diskutierten. Die Resultate wurden verdichtet und in kurz,mittel- und langfristige Szenarien geclustert. In der anschließenden Diskussion ordnen wir die Kernpunkte, die über die Szenarienbeschreibungen hinausgehen, in den wissenschaftlichen Kontext ein. Abschließend werden die Grenzen aufgezeigt und eine Empfehlung für die weitere Forschungsarbeit in diesem Gebiet gegeben. Basierend auf den entwickelten Zukunftsbildern werden Implikationen für die Verkehrsunternehmen abgeleitet und die Relevanz der Haltestelle für unsere Gesellschaft ermittelt.

\section{Fachlicher Hintergrund}

Innovative Entwicklungen beim ÖPNV sind derzeit vor allem in Form von Mobility-as-a-Service-Konzepten zu finden. Seit einigen Jahren werden in verschiedenen Städten und Kommunen MaaS-Konzepte entwickelt und erprobt (Jittrapirom et al. 2017). Mobility-as-a-Service ist als ein neuartiges innovatives Verkehrskonzept zu verstehen, das verschiedene Verkehrsmittel wie den ÖPNV, Taxis, Bike- und Carsharing etc. kombiniert, um den Nutzern eine maßgeschneiderte, komfortable und integrierte Mobilitätslösung anzubieten (Hietanen 2014). Ziel von MaaS ist es, diese kundenzentrierten Mobilitätslösungen so attraktiv und komfortabel zu gestalten, dass Nutzer auf ein Privatfahrzeug weitgehend verzichten können (Jittrapirom et al. 2017; Kamargianni und Matyas 2017). Dafür werden dem Nutzer auf Basis von IoTDaten individuelle und flexible Routen mit nahtlos ineinandergreifenden Verkehrsmitteln angeboten, die er über eine integrierte Schnittstelle (i.d.R. eine App) planen, buchen und bezahlen kann. So ist eine einfache, automatisierte pay-per-use-Abrechnung und Bezahlung für die Nutzer möglich. In den existierenden MaaS-Projekten 
werden jedoch auch häufig Monatsabonnements zu einem festen Preis angeboten (z. B. „Mobility Shop“ Hannover, Deutschland; „UbiGo“ Gothenburg, Schweden).

In den letzten Jahren haben sich Transportplaner, Wissenschaftler und andere Experten intensiv mit den Potentialen und Folgen von MaaS auseinandergesetzt. Im Zuge der Idee, Transport als flexible Tür-zu-Tür-Dienste anzubieten, bei denen der Nutzer einen Startort frei wählen kann, wird das dynamische Routing von Bussen diskutiert. Bereits seit den 1970er Jahren existieren Ansätze zur digitalen Routenoptimierung, u.a. das von Newton und Thomas (1969) entwickelte Modell zur computergestützten Routenplanung von Schulbussen angesichts gegebener Stopppunkte. In diesem Rahmen befasst sich die Forschung auch mit Modellen zum bewussten Überspringen von Haltestellen (Liu et al. 2013; Chen et al. 2015). Heute ist neben der Routenplanung vor allem die Angabe von Fahrplänen in Echtzeit über dynamische Fahrgastinformationen an Haltestellen von Bedeutung für die Fahrgäste (Monzon et al. 2013). Die Qualität und Zugänglichkeit von Informationen hat einen kritischen Einfluss auf die Entscheidung potentieller Fahrgäste für oder gegen die Nutzung des ÖPNVs (Tavares et al. 2015). MaaS-Konzepte mit dynamischem Routing könnten die feste Haltestelle in Zukunft obsolet machen, wenn der Nutzer einen Bus bedarfsorientiert bestellt und ein optimaler Einstiegsort mittels Algorithmen berechnet wird (Hensher 2017). Die vorgestellten MaaS-Studien thematisieren jedoch nicht, welche Folgen sich für die festen Bushaltestellen vor dem Hintergrund zunehmend dynamischen Routings der Busse ergeben.

Vielmehr befasst sich die Literatur rund um die Bushaltestelle insbesondere mit der optimalen Platzierung von Haltestellen im urbanen Raum sowie ihrer Wirkung auf Personen, Verkehr, und bebaute Umgebung. Die optimale Platzierung von Haltestellen kann z. B. nachfrageorientiert erfolgen (Tirachini 2014), anhand der strukturellen Gegebenheiten (Chen et al. 2015) oder unter Berücksichtigung der sozialen Kosten, bestehend aus den Kosten der Nutzer und den Kosten des Betreibers (Ibeas et al. 2010). Wie intensiv eine Haltestelle genutzt wird ist abhängig von der Umgebung, in der sie platziert ist. Bei der Platzierung von Haltestellen müssen daher auch die topographischen Gegebenheiten berücksichtigt werden (Ceder et al. 2015). Auch Chakour und Eluru (2016) stellen bei ihrer Analyse der Einflussfaktoren auf das Fahrgastaufkommen Faktoren, wie an die Haltestelle angrenzende Parks, Schnellstraßen oder Firmen in den Vordergrund (Chakour und Eluru 2016).

Choi et al. (2018) befassen sich mit den ökologischen und gesundheitlichen Auswirkungen der Platzierung von Haltestellen in der Nähe von Kreuzungen und berücksichtigen dabei vor allem die Feinstaubbelastung der an den Haltestellen wartenden Personen. Im Hinblick auf Verbrechen an Haltestellen sind die tatsächliche und die wahrgenommene Sicherheit bei der Entwicklung der bebauten Umgebung (Loukaitou-Sideris 2006), speziell der Haltestelle (Liggett et al. 2001), zu berücksichtigen. Abseits der bebauten Umgebung ist zu beachten, dass eine steigende Anzahl von Busstopps an Haltestellen die Gesamtreisezeit erhöht und sich somit ebenfalls auf die Entscheidung potentieller Fahrgäste auswirken kann (Tirachini et al. 2014).

Umgekehrt kann sich die Haltestelle auch auf ihre bebaute Umgebung auswirken. Bei der Auswahl von Haltestellen müssen deshalb auch die Auswirkungen von Standort und Design auf die Effektivität des Individualverkehrs berücksichtigt werden. Die Platzierung von Haltestellen vor oder nach Kreuzungen kann Verzöge- 
rungen für nachfolgende Autos und Busse verursachen (Gu et al. 2014). Die immer häufiger mit Elektromotoren betriebenen Busse lassen den Haltestellen eine weitere Funktion zukommen. Schnellladebusse können Haltestellen als Ladestation im laufenden Betrieb nutzen (Rogge et al. 2018).

\section{Methodik}

Zur Beantwortung der Forschungsfrage führten wir qualitative Interviews mit acht Fachexperten durch. Die Methode der qualitativen Interviews ist ein offenes Verfahren, das die Teilnehmer zur ausführlichen Darstellung ihrer eigenen Weltsicht bzw. Systemrelevanz anregt (Kohli 1978). Der Verlauf der qualitativen Interviews basiert auf einem Fragenkatalog, der von den acht Fachexperten um eigene innovative Ideen ausgeweitet wurde. Der vollständige Interviewleitfaden ist als Anhang online verfügbar (Onlinematerial 1). Ziel der qualitativen Interviews ist es, das Wissen der unterschiedlichen Fachexperten zusammenzuführen, um zukünftige Ereignisse, Trends, technische Entwicklungen und dergleichen möglichst gut einschätzen zu können. Aus den unterschiedlichen Ergebnissen der Befragungen haben wir vor dem Hintergrund von MaaS-Konzepten Zukunftsvisionen für die Bushaltestelle abgeleitet.

Die Auswahl der Fachexperten erfolgte mit dem Ziel fundiertes Know-how aus unterschiedlichen Bereichen - Fahrgastinformationssysteme, Infrastruktur, Betrieb und Marketing der Verkehrsbetriebe - zu erhalten, um möglichst diverse Aspekte und Betrachtungsweisen zu erfassen. Bei der Auswahl der Fachexperten wurde darauf geachtet, dass es sich um Fachexperten aus Großstädten unterschiedlicher Regionen der Bundesrepublik Deutschland handelt. Bei den Fachexperten handelt es sich ausschließlich um Führungskräfte des oberen bzw. Topmanagements deutscher Nahverkehrsunternehmen, die in ihrer strategischen und operativen Beschäftigung unter anderem im Bereich der Haltestelle tätig sind. Bei den durchgeführten Interviews konnte die Expertise von Entscheidern u.a. der vier deutschen MillionenMetropolen berücksichtigt werden.

Tab. 1 gibt Auskunft über die befragten Manager sowie über die Größe der jeweils von dem Nahverkehrsunternehmen betriebenen Stadt.

Die gewählte Methode sind leitfadengestützte, teiloffene Interviews, die in sieben der acht Fällen - aufgrund der Distanz - telefonisch und in einem Fall vis a vis geführt werden konnten. Der Fragenkatalog (siehe Onlinematerial 1) wurde den Fachexperten vor dem jeweiligen Gespräch zur Verfügung gestellt, um weitere Gesprächsbereiche zu ermöglichen. Im Rahmen der einzeln geführten Interviews haben wir zunächst allgemein die Themenfelder Digitalisierung, multimodale Haltestelle und Design der Haltestelle auf Grundlage des aktuellen Mobilitätsdiskurses thematisiert. Anschließend haben wir die Megatrends Digitalisierung und innovative Mobilität bezogen auf die zukünftige Haltestelle mit Fachexperten diskutiert. Bei der Beantwortung der Forschungsfrage ist als Annahme ein Bleiben, Verändern oder Verschwinden der Haltestelle möglich. Die Fachexperten wurden gebeten ihr Knowhow in kurz-, mittel- und langfristige Entwicklungen der Haltestelle in Szenarien zu beschreiben und zu analysieren. Die Interviews dauerten zwischen 48-95 min Alle 
Tab. 1 Übersicht über die Experten

\begin{tabular}{|c|c|c|c|c|c|c|}
\hline Experte & Position & Aufgabenbereich & Geschlecht & Alter & $\begin{array}{l}\text { Anzahl der Ein- } \\
\text { wohner }\end{array}$ & $\begin{array}{l}\text { Dauer des } \\
\text { Interviews } \\
\text { in Std }\end{array}$ \\
\hline 1 & $\begin{array}{l}\text { Fachbereichs- } \\
\text { leiter }\end{array}$ & $\begin{array}{l}\text { Fahrgastinforma- } \\
\text { tionssysteme }\end{array}$ & $\mathrm{m}$ & $40-50$ & $600.000-700.000$ & $01: 35$ \\
\hline 2 & $\begin{array}{l}\text { Fachbereichs- } \\
\text { leiter }\end{array}$ & $\begin{array}{l}\text { Fahrgastinforma- } \\
\text { tionssysteme }\end{array}$ & $\mathrm{m}$ & $50-60$ & $1.000 .000-1.100 .000$ & $01: 34$ \\
\hline 3 & $\begin{array}{l}\text { Fachbereichs- } \\
\text { leiter }\end{array}$ & $\begin{array}{l}\text { Fahrgastinforma- } \\
\text { tionssysteme }\end{array}$ & $\mathrm{m}$ & $30-40$ & $300.000-400.000$ & $01: 23$ \\
\hline 4 & $\begin{array}{l}\text { Geschäfts- } \\
\text { führer }\end{array}$ & $\begin{array}{l}\text { Kaufmännische } \\
\text { Geschäftslei- } \\
\text { tung }\end{array}$ & $\mathrm{m}$ & $40-50$ & $1.800 .000-1.900 .000$ & 01:06 \\
\hline 5 & $\begin{array}{l}\text { Geschäfts- } \\
\text { führerin }\end{array}$ & $\begin{array}{l}\text { Kaufmännische } \\
\text { Geschäftslei- } \\
\text { tung }\end{array}$ & $\mathrm{w}$ & $50-60$ & $300.000-400.000$ & $00: 48$ \\
\hline 6 & Bereichsleiter & Infrastruktur & $\mathrm{m}$ & $50-60$ & $500.000-600.000$ & 01:01 \\
\hline 7 & $\begin{array}{l}\text { Fachbereichs- } \\
\text { leiter }\end{array}$ & $\begin{array}{l}\text { Angebots- } \\
\text { grundlagen }\end{array}$ & $\mathrm{m}$ & $30-40$ & $3.600 .000-3.700 .000$ & 01:00 \\
\hline 8 & $\begin{array}{l}\text { Fachbereichs- } \\
\text { leiter }\end{array}$ & Verkehrstelematik & $\mathrm{m}$ & $40-50$ & $1.500 .000-1.600 .000$ & 01:00 \\
\hline
\end{tabular}

Interviews wurden aufgezeichnet, anschließend transkribiert und themenanalytisch ausgewertet.

Für die inhaltliche Analyse des Datensatzes folgten wir einem thematischen Analyseverfahren auf Basis von Braun und Clarke (2006). Die thematische Analyse zeigt Gemeinsamkeiten und Unterschiede zwischen den Datensätzen auf. Sie ermöglicht auch die Generierung unvorhergesehener neuer Erkenntnisse. Sie wird für die Analyse qualitativer Daten verwendet und ist flexibel, da es an keinen theoretischen Rahmen gebunden ist. Für unsere Analyse haben wir uns zunächst mit dem Material vertraut gemacht, bevor wir Themen und Unterthemen kodierten. Das iterativ entwickelte Codesystem wurde auf alle Interviews angewendet. Im Folgenden Kapitel stellen wir die analytischen Ergebnisse dar.

\section{Ergebnisse}

Sämtliche in den drei Szenarien beschriebenen Einschätzungen und Entwicklungen beruhen auf den Aussagen der Fachexperten. So besteht unter den Experten Einigkeit, dass sich das Umfeld der Haltestelle und die Rahmenbedingungen generell verändern. Der demografische Wandel zeigt, dass mit einer steigenden Lebenserwartung zu rechnen ist und immer mehr Menschen durch ein gutes Gesundheitssystem bis ins hohe Alter agil bleiben. In Zukunft wird die Urbanisierung weiter Bestand haben und die Menschen bevorzugen ein Leben in den Großstädten. Die daraus resultierende wachsende städtische Bevölkerung führt zu einer stärkeren Nachfrage nach Mobilität. Vor dem aktuellen Diskurs in Deutschland gibt es bereits einige Innenstädte, in denen ein Dieselfahrverbot besteht. Es ist davon auszugehen, dass 
es aufgrund steigender Emissionswerte zu noch weiteren Einschränkungen kommen muss. Diese Auflagen schwächen den Individualverkehr, der den stärksten Konkurrenten des ÖPNV darstellt. Die Menschen sind interessierter an Angeboten der kollektiven Beförderung, wenn es ausgereifte, flächendeckende Mobilitätsketten gibt. Seit Einführung der europäischen Datenschutz-Grundverordnung sind die Nutzer kritischer im Umgang mit ihren Daten und gehen sparsamer damit um. Bei einer langfristigen Realisierung vom autonomen Fahren wird die Thematik neu zu diskutieren sein. Vor dem Hintergrund dieser Rahmenbedingungen schätzen die Fachexperten die Zukunft der innerstädtischen Bushaltestelle wie folgt ein.

\subsection{Kurzfrist-Szenario: Erwartete Veränderungen der Haltestelle}

\subsubsection{Digitalisierung}

Echtzeit-Informationen Die Bushaltestelle ist in Städten deutschlandweit ähnlich gestaltet und weist einen hohen Wiedererkennungswert für die Bevölkerung auf. Trotz der Weiterentwicklung der digitalen Infrastruktur werden nach Expertenprognosen kurzfristig Fahrplaninformationen weiterhin in Papierform zur Verfügung stehen, da gesetzliche Vorgaben verlangen, dass der Kunde auch jenseits von digitalen Medien erreicht werden muss. Unter den Experten besteht jedoch Einigkeit, dass ein weiterer Ausbau der digitalen und IT-gestützten Infrastruktur eine papierlose Haltestelle perspektivisch möglich macht. Hier nennen die Experten vor allem zwei zentrale Ziele: Erstens, die Digitalisierung der Fahrgastinformationen, insbesondere des Fahrplans, auf die der Fahrgast sowohl an der Haltestelle als auch über eine App zugreifen kann sowie zweitens die ökologisch effiziente, „grüne“ Haltestelle.

Allen Fahrgästen muss auch weiterhin gleichberechtigt ein Zugang zu Fahrgastinformationen gegeben sein. Echtzeit-Fahrgastinformationen sind an den Haltestellen vor Ort für jede Zielgruppe von Nutzen. Es kann jedoch nicht jeder Kunde auf digitale Informationen zugreifen, daher werden Fahrgastinformationen weiterhin über unterschiedliche Kanäle bereitgestellt - neben digital verfügbar gemachten Informationen über Reiseinformationssysteme auch in Form von Papierfahrplänen. Gerade die vielen älteren Bürger, die in den Innenstädten den ÖPNV nutzen, erreichen digitale Medien nicht. An Bushaltestellen muss es dieser großen Zielgruppe möglich sein, auch ohne Smartphone eine optimale Informationsversorgung zu erhalten.

Laut Experten werden die Haltestellen kurzfristig flächendeckend mit digitalen Abfahrtsmonitoren (,DFI-Lights“) ausgestattet, die dynamische Fahrgastinformationen in Echtzeit für den Kunden gut sichtbar anzeigen. Dem Kunden werden über diese Monitore alle für die Fahrt relevanten Informationen angezeigt, sodass er sich an den Haltestellen keiner weiteren Informationsquelle zu bedienen braucht. Der Einbau in das Beschilderungssystem der Haltestelle ist mit wenig Aufwand und finanziellen Mitteln umsetzbar. Des Weiteren ist keine Erweiterung der Stromzuleitungen notwendig, da diese Abfahrtsmonitore mit Batterien bzw. Solarmodulen betrieben werden können. Die bestehende IKT-Infrastruktur kann durchgängige Echtzeitinformationen über Abfahrten der Busse gewährleisten.

Darüber hinaus ist es möglich über das Intermodal Transport Control System (ITCS) Zusatzinformationen, wie z. B. bevorstehende Veranstaltungen oder Ergeb- 
nisse von Weltmeisterschaftsspielen, für die Fahrgäste an den Haltestellen ergänzend zur Fahrplanauskunft anzuzeigen. Es muss jedoch sichergestellt werden, dass diese Zusatzinformationen nicht den eigentlichen Zweck der Abfahrtinformation negativ beeinflussen.

Ein Add-on zu den Fahrgastinformationen bilden Navigationssysteme, die Anschluss-Beziehungen darstellen. Beim Umsteigen braucht der Kunde zielführende Orientierungshilfen. Nach dem Verlassen des Fahrzeuges muss eine schnelle Orientierung durch effektive Beschilderung an den Haltestellen in digitaler und gedruckter Form möglich sein. Die Haltestellenschilder bieten hierbei statische Information, die durch dynamische Realtime-Informationen an den Monitoren ergänzt werden. Die digitalen Medien sollen dem Fahrgast einen zeitökonomischen Ein- bzw. Umstieg ermöglichen.

Neben den Fahrgastinformationen an den Haltestellen sind Apps als Informationsmedium für den Kunden entscheidend. Die Verkehrsunternehmen stellen in ihren Apps Echtzeit-Informationen zur Verfügung. Der Kunde kann über sein Smartphone die notwendigen Abfahrtinformationen für sein Fahrzeug ortsunabhängig abrufen. Der Abfahrtzeitpunkt des Fahrzeuges ist für den Kunden schon vor der Ankunft an der Haltestelle bekannt und kann so zu einer reduzierten Wartezeit an den Haltestellen führen.

Um einen ökologischen Mehrwert zu generieren, ist der Wandel zu einer „grünen“ Haltestelle vorgesehen. Die Kosten für teure Infrastruktur und Wartung von LCDTechnik werden durch den Nutzen der neuen e-Paper Technik reduziert. Eine grüne Haltestelle funktioniert überwiegend durch Nutzung von regenerativen Stromversorgungsmöglichkeiten, wie Solarpanels und umweltfreundlichen, batteriebetriebenen Anzeigen. An größeren Umsteigepunkten der Bushaltestellen, besonders an Schnittpunkten zum Bahnverkehr, sind die großflächigen LCD-Anzeiger weiterhin für das Umsteigemanagement hilfreich.

IKT-gestütztes Marketing Die Experten bestätigen, dass grundsätzlich eine technologische Ausstattung der Haltestellen mit schnellem WLAN-Zugang möglich ist, jedoch regional unterschiedlich behandelt wird.

Es gibt Großstädte, die kurzfristig WLAN flächendeckend für die private Nutzung an den Haltestellen einführen werden. Das Angebot eines kostenlosen Internetzugangs wird als großer Zusatznutzen für den Kunden bewertet. Durch den WLANZugang dient die Haltestelle als Marketinginstrument der Verkehrsunternehmen und kann Kunden zielgerichteter, individueller, dem Bedarf des Kunden entsprechender Werbung zuspielen. Es ergeben sich weitere Möglichkeiten das Unternehmen als Marke darzustellen. Über WLAN kann dem Kunden beispielsweise Informationen zu seiner bevorstehenden Fahrt vorab gegeben werden. Es könnte ein Profil des Busfahrers, unter Berücksichtigung der Regelung der neuen europäischen Datenschutz-Grundverordnung sowie spezifische Fahrzeugdaten angezeigt werden, um eine persönliche Bindung aufzubauen.

Andere Städte werden nur dann einen WLAN-Zugang an den Haltestellen zur Verfügung stellen, wenn ein effizientes Geschäftsmodell dahintersteht. Es müssen Kooperationen mit anderen Geschäftspartnern verhandelt werden, um eine Finanzierungsmöglichkeit über private Unternehmen sicherzustellen. Eine Möglichkeit 
ist, einen externen Dienstleister mit der Betrauung der Haltestellen zu beauftragen. An zentralen Haltestellen, an denen ein hohes Aufkommen von Touristen alltäglich ist, sowie an Haltestellen mit direkter Anbindung an einen Flughafen entsteht ein hoher Mehrwert durch den WLAN-Zugang. Ein Experte befürchtet, dass ein offenes WLAN sozial schwache Gruppen an die Haltestelle lockt und Kosten für Vandalismus erhöht werden könnten.

Durch die WLAN-Verbindungen findet zwischen den Haltestellen und Fahrzeugen eine noch engere Vernetzung statt. Es können Kapazitäten innerhalb der Fahrzeuge ermittelt werden, die dem Kunden anzeigen, wie hoch das aktuelle Fahrgastaufkommen im Bus ist. Bei zu hoher Auslastung erfolgen Vorschläge über Alternativrouten oder -fahrzeuge. Eine weitere technische Innovation sind USB-Schnittstellen, die dem Kunden eine Lademöglichkeit für Smartphones anbietet.

\subsubsection{Multimodale Haltestelle}

Kurzfristig, so die einheitliche Expertenmeinung, wird es keine flächendeckenden virtuellen Haltestellen geben, und speziell für den Personenmassenverkehr werden in den Innenstädten die Bushaltestellen als feste Punkte im Stadtbild bleiben. Lediglich in innovativen deutschen Großstädten wird es bereits in naher Zukunft virtuelle Haltestellen geben, die als „on demand“-Verkehre durch Drittanbieter ergänzend zum klassischen ÖPNV angeboten werden. Die Experten glauben nicht, dass die deutschen Verkehrsbetriebe diese virtuellen Haltestellen einrichten und betreiben werden. Speziell Tochterunternehmen aus europäischen Automobilkonzernen haben sich auf das neue Geschäftsmodell fokussiert. Diese innovativen „Shuttle on demand"-Systeme sind in Pilotprojekten getestet und werden als smarte, intelligente Transportsysteme sukzessive erweitert.

Das Zukunftsthema Elektromobilität bietet zwei wesentliche Vorteile. Zum einen ist es dem Betrieb möglich die E-Busse an den Haltestellen zu laden und zum anderen Ladestationen für E-Autos von ÖPNV-Kunden an ,,park and ride“-Parkplätzen anzubieten. Die Experten sehen Möglichkeiten die Haltestellen speziell in den Außenbereichen verstärkt mit durch erneuerbare Energien betriebene Ladevorrichtungen auszubauen, die mit entsprechender ,grünen“ Energie durch Solarpanels betrieben wird. Die Realisierung bedarf eines städtebaulichen Konzepts für die benötigten Flächen.

\subsubsection{Design der Haltestellen}

Die Experten glauben, dass die Haltestellen kurzfristig nur kleine optische Veränderungen erfahren und weiterhin fester Bestandteil in den Innenstädten bleiben. Das zunehmende Fahrgastaufkommen ist unter Sicherheitsaspekten zu betrachten und die optimalen Haltestellenabstände dementsprechend anzupassen.

Eine technische Zusatzoption sind Beacon für sehbehinderte Kunden an den Haltestellen, die es möglich machen Fahrgastinformationen vorzulesen. Diese Beacon sind bereits heute an einigen Haltestellen verfügbar und werden bei Umbaumaßnahmen fester Bestandteil der Haltestellenrekonstruktion, um einen optimierten Einstieg zu gewähren. 
Die größte Veränderung wird es kurzfristig hinsichtlich der Bezahlsysteme geben. An vielen Haltestellen gibt es derzeit Fahrscheinautomaten, die hohe Kosten in der Betriebsinfrastruktur verursachen. Kurzfristig werden diese Fahrscheinautomaten aus den Großstädten sukzessive verschwinden. Fahrgäste müssen ihre Tickets zukünftig ausschließlich mit modernen Bezahlsystemen (z. B. per App) kaufen bzw. im Bus. Das Ziel von modernen Bezahlsystemen ist ein vereinfachtes Verfahren für die Kunden und der Abbau von Zugangshemmnissen.

\subsection{Mittelfrist-Szenario: Erwartete Veränderungen der Haltestelle}

\subsubsection{Digitalisierung}

Echtzeit-Information Die Haltestelle der Innenstadt wird mit den gleichen Grundfunktionen bestehen bleiben. Die Fahrpläne werden ausschließlich in digitaler Form angeboten und gepflegt. Mittelfristig wird eine Ausweitung von e-Papier-Lösungen an den grünen Haltestellen auch in den Randgebieten erfolgen, sodass der Fahrplan in Papierform nicht mehr notwendig sein wird. Es ist denkbar, dass die Abfahrtzeiten an die Wände der Haltestellen projiziert werden und so für den Kunden gut lesbar erscheinen. Das Umsteigemanagement erfolgt über die digitale Infrastruktur und gibt konsistente Informationen zu Umleitungen, Störungen und Richtungsänderungen bekannt.

Nach Expertensicht bieten besonders Realtime-Informationen auf Basis von GPSDaten der Busse durch eine hohe Aktualität einen Mehrwert für die Kunden. Demnach ist davon auszugehen, dass die Verkehrsbetriebe mittelfristig die GPS-Daten der Fahrzeuge für die Kunden in der App sichtbar machen. Durch die Transparenz ist es für den Kunden möglich eine visualisierte Standortbestimmung der gewünschten Busse vorzunehmen. Diese technische Erweiterung ist für die Routenplanung von ortskundigen Kunden besonders hilfreich. Der Kunde kann Ampelsysteme und Stauverhalten nach seinen individuellen Erfahrungen einschätzen. Er kann autark entscheiden, zu welchem Zeitpunkt er Richtung Haltestelle aufbrechen muss, um den Bus ohne lange Wartezeiten zu erreichen.

Auf Basis des interaktiven Liniennetzplans wird der IT-Support der Verkehrsbetriebe eine Filterfunktion programmieren, die zur Übersichtlichkeit eine Auswahl von einzelnen Linien und Zeiten ermöglicht.

IKT-gestütztes Marketing Mittelfristig sehen die Experten die Möglichkeit, für die Millionenstädte, die kurzfristig eine flächendeckende Ausstattung der Haltestelle mit WLAN vorgenommen haben, ein digitales, individualisiertes Marketing über die jeweils angebotenen Apps zu steuern. Dadurch kann das Verkehrsunternehmen zielgerichtet Werbeinhalte an den Kunden übermitteln. Es kann haltestellenspezifische Werbung für die umliegenden Geschäfte und deren Rabattaktionen geschaltet werden. Auch Verbraucherinformationen und Präferenzen der Kunden werden beim Routing der Werbeblöcke berücksichtigt. Eine Veräußerung dieser Daten oder Nutzung zu Forschungszwecken, unter Berücksichtigung der Inhalte der neuen EUDatenschutzgrundverordnung, ist denkbar. Im Rahmen des ganzheitlichen, modernen Haltestellenmanagements können - neben den Werbemaßnahmen über die an- 
gebotenen Apps - Werbespots an den digitalen Fahrzeitanzeigen Bestandteil des Haltestellen-Entertainmentprogramms werden.

\subsubsection{Multimodale Haltestelle}

Auch in über fünf Jahren prognostizieren die Fachexperten keinen radikalen Wandel der klassischen Haltestelle. Die klassische Haltestelle bleibt in den noch dichter besiedelten Innenstädten weiterhin die zentrale Anlaufstelle für Personenmassenverkehre. Der Bedarf an „on demand“-Verkehren wird zunehmen, jedoch nur in geringem Ausmaß. Diese Form der Personenbeförderung erfolgt als Zubringer für Gebiete, die nicht den direkten Zugang zum ÖPNV genießen und auf MaaS als Mobilitätslösung angewiesen sind.

Daneben werden virtuelle Haltestellen ergänzend zur klassischen Haltestelle bei Gelegenheitsverkehren und großen Events vereinzelt angeboten, nicht jedoch als Option für den alltäglichen Personenverkehr. Es gibt mittelfristig einen ausgebauten kollektiven Personenverkehr, ergänzt um weitreichende MaaS-Konzepte. Die Fahrgastzahlen an der klassischen Haltestelle sind und bleiben jedoch deutlich höher als an den virtuellen Haltestellen.

Es gibt ein MaaS-Konzept, das aufgrund von quantitativen und qualitativen Datensätzen genaue Prognosen zu den nächstgelegenen ergänzenden Mobilitätsmöglichkeiten (wie Fahrradverleihsysteme, E-Scooter oder Car-Sharing-Modelle) abgibt.

\subsubsection{Design der Haltestellen}

Die Fachexperten sehen mittelfristig eine Stärkung der klassischen Haltestelle als realistisch an. Demnach erfolgt ein massiver Ausbau der bestehenden zentralen Haltestellen in den Innenstadtbereichen, aber auch eine punktuelle Funktionserweiterung von Haltestellen an hochfrequentierten Randbereichen. Hieraus entstehen vermehrt Kooperationen mit Unternehmen speziell aus den Bereichen Dienstleistung, Logistik und Einzelhandel, die sich die Haltestelle als Vertriebsinstrument zu eigenen machen. Ein Wäscheservice als externer Dienstleister an den Knotenhaltestellen ermöglicht besonders viel beschäftigten Kunden flexibel ihre BusinessKleidung komfortabel reinigen zu lassen. Durch einen individualisierten Pin-Code sind die Abgabe sowie das Abholen zu jeder Tages- und Nachtzeit flexibel gestaltbar.

Ein strategisch angelegtes Logistikkonzept zur Positionierung von Packstationen an den Haltestellen entlastet die Zusteller und vereinfacht den komplexen Lieferverkehr. Die bestehende Infrastruktur der Verkehrsunternehmen gibt dem Kunden die Flexibilität Pakete nach seinen zeitlichen Ressourcen abzuholen, ohne Öffnungszeiten beachten zu müssen. Das Retournieren von ungewünschten Produkten kann ebenfalls durch diese Packstationen wahrgenommen werden.

Neben diesen dienstleistenden und logistischen Veränderungen sorgen sogenannte Smart Walls für ein exklusives Einkaufserlebnis und als Schnittstelle zum Einzelhandel. Der Kunde kann bequem an der Haltestelle seinen Warenkorb zusammenstellen und die Produkte an den vorgesehenen Terminals bestellen. Die Waren werden fachmännisch, unter Berücksichtigung von Kühlung, Hygiene etc., in die Boxen an den Haltestellen gepackt und sind mit einem über App zugeschickten Code verfügbar. 
Nicht verderbliche Produkte können vorrätig in den Haltestellen gelagert und gegen Bezahlung sofort ausgegeben werden.

Eine Bündelung dieser modernen Komponenten kann in Form eines Use-StoreRaums erfolgen, der dem Kunden einen bequemen und witterungsunabhängigen Aufenthalt ermöglicht. Dieser Use-Store-Raum führt weitere Innovationen zusammen. Über Flat Screens innerhalb dieser Use-Store-Räume wird ein Entertainmentprogramm mit Kurzclips und Nachrichten sowie ein Ausbau mit Playstations angeboten. Die Haltestelle ist Teil eines Erlebnisses. An zentralen Knotenpunkten werden Marketingmaßnahmen mit Events wie Show-Cooking verbunden.

\subsection{Langfrist-Szenario: Erwartete Veränderungen der Haltestelle}

\subsubsection{Digitalisierung}

Echtzeit-Information Die Echtzeitinformationen sind ausgereift und stellen eine konsistente Komponente dar. Die Echtzeit wird über diverse digitale Infrastrukturen angezeigt. Die Berechnung der Realtime erfolgt über Abstandsanzeigen, die mittels detaillierten GPS-Informationen der Busse, aber auch unter Berücksichtigung von Verkehrsmetadaten aus dem Individualverkehr, dem Kunden eine genaue Ankunftszeit vorgeben. Für die Fahrgäste gibt es eine visualisierte Darstellung des Streckenverlaufs und der zur Verfügung stehenden Mobilitätsoptionen. Eine exakte Standortbestimmung der Fahrzeuge gepaart mit den Einschätzungen der Kunden führt zu einer optimalen Routengestaltung. Dieses Routing umfasst langfristig auch die Wege des Individuums vom jeweiligen Standort bis zur nächstgelegenen Haltestelle. Das Smartphone bildet den Zugang zur digitalen Infrastruktur und zeigt eine virtuelle Umgebung, die direkt zur Haltestelle führt. Veränderungen während der Fahrt werden direkt im Routing berücksichtigt und führen zu einer optimierten Streckenführung. Der Bestimmungsort bleibt bei der Navigation konstant und alle Mobilitätsdienste richten sich nach dem Fahrtziel.

IKT-gestütztes Marketing Langfristig wird aus Sicht der Experten der defizitäre ÖPNV verstärkt auf finanzielle Entlastungen angewiesen sein und dementsprechend auch Einnahmen aus Werbemaßnahmen und Events an den Haltestellen generieren. Speziell die Verlagerung von Werbemaßnahmen auf die individuellen Endgeräte der Kunden bringt finanzielle Mittel und führt zu vermehrten Ressourcen für Fahrgastinformationen.

Aufgrund der fehlenden Rentabilität erwarten die Experten, dass sich langfristig neue Betreibermodelle für entlegene Haltestellen entwickeln. Große Unternehmen, wie z. B. Möbelhäuser, Baumärkte oder Einzelhandelsketten, kommen als Betreiber einer eigenen Haltestelle infrage, die mit dem Betrieb das Ziel verfolgen können den Zugang zum Unternehmen zu gewährleisten und neue Kunden zu akquirieren. Die Nahverkehrsunternehmen könnten dann eine Kostenpauschaule für das Anfahren der Haltestelle entsprechend der vereinbarten Taktung erhalten. Diese Haltestellenbezeichnungen dienen als Orientierungshilfe und können vom jeweiligen Betreiber benannt werden. 


\subsubsection{Multimodale Haltestelle}

Die Experten sind sich einig, dass langfristig das autonome Fahren realisiert wird und den ÖPNV entsprechend beeinflusst. Durch die ausgereifte Selbstfahrtechnik entstehen viele Möglichkeiten der innovativen Personenbeförderung. Für eine bedarfsgerechte Mobilität können beispielsweise kleine autonome Shuttlebusse die Kunden aus ländlichen Regionen ,on demand“ an die Personenmassenverkehre anbinden und einen flexiblen ÖPNV als Fundament von MaaS sicherstellen.

Ein radikaler Wegfall der Haltestellen führt zu einer erhöhten Flexibilität für den einzelnen Kunden, führt jedoch auch zu höheren Wartezeiten, wenn der Bus von der planmäßigen Route abweicht und sich dadurch die Fahrtzeit verlängert. Jede ,on demand“-Fahrt ist individualisiert und erfordert ein exaktes Fahrtmanagement. Nach vorherrschender Expertenansicht ist deshalb auch langfristig nicht mit „on demand“-Verkehren als alleinige Form der Mobilität zu rechnen. Die Personenmassenverkehre in den Innenstadtbereichen werden weiterhin durch die festen Haltestellen abgedeckt.

\subsubsection{Design der Haltestellen}

Nach der Bündelung von Innovationen und dem massiven Ausbau der bestehenden zentralen Haltestellen ergeben sich nach Experteneinschätzung langfristig weitere Möglichkeiten speziell aus den Bereichen der demografischen Entwicklung, dem autonomen Fahren und der Videoüberwachung.

Nach wie vor steht der Mensch im Mittelpunkt der Mobilität und die Haltestelle dient als Ort der Verbindung. Die im langfristigen Szenario älteren Generationen kommen mit den Funktionen des Smartphones zurecht, da sie bereits damit aufgewachsen sind. Vor diesem Hintergrund besteht ein Mehrbedarf an Pflegekräften und sozialen Einrichtungen, die im langfristigen Szenario an den Haltestellen als Unterstützer gebucht werden können. Die Mobilitäts-App bietet Funktionen an einer Haltestelle einen Helfer anzufragen bzw. sich als freiwilliger Helfer zu registrieren.

Die Fachexperten rechnen fest mit einer flächendeckenden Einführung von autonom fahrenden Bussen. Die Veränderungen durch diese neue Technik werden auch eine Veränderung an der Haltestelle notwendig machen. Eine Vermessung der Abstände, der Höhe zum Ein- und Aussteigen sowie die Bordsteinkanten folgen der Technik.

Durch eine umfassende Videoüberwachung an den Haltestellen werden Straftaten und Vandalismus festgehalten. Die Aufzeichnungen an den Haltestellen dienen als Beweismittel und sollen dem Kunden ein Gefühl der Sicherheit vermitteln.

\section{Diskussion und Schlussfolgerung}

Die Haltstelle ist über Jahrzehnte hinweg als Gesicht der Verkehrsunternehmen in der Öffentlichkeit bekannt und hat wenig Veränderungen erfahren. In Zeiten eines zunehmenden Digitalisierungsgrads und moderner MaaS-Konzepte wird auch die Haltestelle modernisiert. 
Nach Fachexpertensicht sind bereits kurzfristig Veränderungen der Bushaltestelle in den Innenstädten realistisch. Im Themenfeld der Digitalisierung werden EchtzeitInformationen deutschlandweit und flächendeckend verfügbar sein und um moderne Kunden-Navigationssysteme ergänzt. Durch schnellen WLAN-Zugang kann ein kundenspezifisches Marketing sowie eine enge Vernetzung zwischen Haltestelle und Fahrzeug erfolgen. In einigen Großstädten finden bereits Tests von „on demand“Verkehren statt, die aus Sicht der Experten jedoch kurzfristig lediglich als Ergänzung zum klassischen ÖPNV zu verstehen sind. Nach vorherrschender Fachexpertenansicht ist weder kurz-, mittel- noch langfristig mit einer Verdrängung der klassischen Haltestelle zugunsten von ,on demand“-Verkehren als alleiniger Form der Mobilität in Großstädten zu rechnen. Diese Überzeugung ergibt sich aus der Abwägung von Kosten und Nutzen flexibler und virtueller Haltestellen. Deren Nutzen ergibt sich für den Fahrgast aus der Tatsache, dass er bedarfsorientiert und unabhängig von Zeit und Ort einen Bus bestellen und dort zu- und aussteigen kann. Mehrwerte ergeben sich aus der Wegersparnis zur oder von der festen Bushaltestelle sowie der individuellen, nicht vom Fahrplan abhängigen, Abfahrtszeit. Die individuelle Bedienung eines jeden Fahrgast würde aufgrund der in Kauf zu nehmenden Umwege für die Verkehrsbetreiber hohe zeitliche und damit auch finanzielle Aufwände bedeuten. Als Kompromiss käme eine Bündelung zeitlich und räumlich korrelierender Nutzeranfragen infrage, die dann an einer Stelle zu einem Zeitpunkt gemeinsam von einem Fahrzeug bedient werden.

Die Personenmassenverkehre in den Innenstadtbereichen werden weiterhin durch die klassischen festen Haltestellen bedient. Dies gilt insbesondere für die Innenstadtbereiche, in denen sich eine Entfernung von durchschnittlich ca. $400 \mathrm{~m}$ zwischen den Haltestellen als besonders effizient erwiesen hat. Das Angebot individueller Ein- und Ausstiege wird auch in Zukunft für die Verkehrsunternehmen nicht kosteneffizient darstellbar sein. Die dichte Besiedlung mit Haltestellen bietet Kunden einen zumutbaren, komfortablen Einstieg in die Busverkehre. Das Taxi als individuelle Lösung, zu höheren Preisen, besteht für das Individuum als Zusatzoption weiterhin.

Unter der Annahme, dass die Prognosen des kurzfristigen Szenarios eingetreten und die klassische Haltestelle sich entsprechend den aufgeführten Maßnahmen verändert hat, sind die Grundlage für die Einschätzungen des mittelfristigen Szenarios. Demnach erwarten die Fachexperten mittelfristig auch weiterhin einen zunehmenden Digitalisierungsgrad. Die Echtzeit-Fahrplaninformationen geben Auskünfte auf Basis von GPS-Daten der Fahrzeuge und können per App für den Kunden mit einer visualisierten Standortbestimmung jederzeit abgerufen werden. An den Haltestellen findet ein zielgruppenspezifisches Haltestellen Entertainment Programm Einzug und durch den Ausbau mit Kooperationspartnern kann die Haltestelle zusätzliche Services anbieten.

Die Vermarktung von Werbeflächen stellt einen finanziell lukrativen Part der Haltestelle dar. Die Herausforderung für Verkehrsunternehmen besteht darin, die Balance zwischen Werbeangeboten und der effektiven Präsentation der Fahrgastinformation zu wahren.

Ungeachtet dessen werden ganzheitliche MaaS-Konzepte weiter in den Fokus der Öffentlichkeit rücken. Ein extremes langfristiges Zukunftsszenario der Haltestelle ist die Abschaffung der klassischen, festen Haltestellen. Eine derartige Mobilitätsland- 
schaft wird stark geprägt von virtuellen Haltestellen, sogenannten „Hotspots“ sein, die jederzeit durch einen Zusammenschluss von mehreren Personen entstehen können.

Während die klassische Haltestelle nach Ansicht der Experten im ländlichen Raum langfristig verschwindet und der Kunde verstärkt bedarfsorientiert über virtuelle Haltestellen befördert wird, wird die stationäre Haltestelle in Innenstadtbereichen auch langfristig eine große Rolle spielen. Auch langfristig gehen die Fachexperten von wachsenden Städten und einer erhöhten Nachfrage von Mobilitätsdienstleistungen aus. Des Weiteren muss sich der ÖPNV auch langfristig mit dem Individualverkehr messen. Die Summe aller Mobilitätslösungen muss für den Kunden als vorteilhaft im Vergleich zum Individualverkehr wahrgenommen werden. Ein eigener PKW bietet das Gefühl einer vermeintlichen Mobilitätsflatrate, allerdings stößt dieses Gefühl von Freiheit schnell an seine Grenzen. Staus und volle Straßen in der Rush Hour sowie Dieselverbote schränken den Individualverkehr stark ein. Hier gilt es durch ein attraktives Mobilitätskonzept anzusetzen und die Verkehrsverlagerung voranzutreiben.

Die befragten Fachexperten rechnen langfristig fest mit einer flächendeckenden Einführung von autonom fahrenden Bussen und sehen hierdurch eine Erneuerung der Haltestelle als unumgänglich. Weitere zukünftige Mobilitätsmöglichkeiten wären autonom betreibbare Drohnen oder Seilbahnen. Speziell Seilbahnen könnten zu einer Entlastung von Straßen führen und Teile der Beförderung in den Luftraum verlagern. Diese Mobilitätsmöglichkeiten benötigen ein innovatives Haltestellemanagement.

Der meist subventionierte Nahverkehr wird verstärkt im Wettbewerb stehen und auch langfristig auf finanzielle Mittel angewiesen sein. Ein Outsourcing von Haltestellen an finanzstarke Kooperationspartner als Betreiber entlastet die Investitionsund Instandhaltungskosten der ÖPNV-Unternehmen und bietet gleichzeitig Ertragsmöglichkeiten durch Generieren von neuen Streckenkursen.

\section{Grenzen und zukünftige Forschung}

Trotz dieser diskutierten Beiträge sind einige Einschränkungen erwähnenswert. Es handelt sich um die Einschätzung von Fachexperten und somit um eine Prognose von zukünftigen Entwicklungen. Die kurz- und mittelfristigen Entwicklungen sind relativ gut absehbar, da diese erwarteten Innovationen überwiegend technisch möglich - jedoch noch nicht marktreif - ausgestaltet sind. Langfristige Entwicklungen können hinsichtlich ihrer Eintrittswahrscheinlichkeit nicht genau prognostiziert werden, da erst vorgelagerte Techniken erfolgreich getestet, umgesetzt und flächendeckend eingeführt werden müssen.

Eine kurzfristige Einführung von flächendeckenden, innovativen Bezahlsystemen bietet den Fahrgästen eine moderne Möglichkeit ein Ticket, über die Grenzen von Verkehrsverbunden hinweg, z. B. per Smartphone zu erwerben. Der gleichzeitige Abbau von Ticketautomaten an den Haltestellen könnte dazu führen, dass der Zugang zum ÖPNV für einzelne Personengruppen erschwert wird. Gerade der ÖPNV als fester Bestandteil der Daseinsvorsorge sollte ohne Zugangsbarrieren für jeden Bürger zugänglich sein und bleiben. 
Durch die neue EU-Datenschutzgrundverordnung sind Kunden sensibilisiert, sorgsam mit ihrer digitalen Persönlichkeit umzugehen. Fake-News und auch steigende Cyberangriffe nehmen verstärkt Einfluss auf Kunden, aber auch auf Verkehrsunternehmen. Besonders gezielte Cyberangriffe auf kritische Infrastrukturen setzen Grenzen in die Möglichkeiten der Digitalisierung und hemmen das Innovationsvermögen der Haltestelle. Des Weiteren gilt es zu beachten, dass die Energieverfügbarkeit und die natürlichen Rohstoffe endlich sind. Sollte die eingesetzten Technik mangels Rohstoffe nicht mehr verfügbar sein, gilt es substituierende Möglichkeiten zu finden.

Präferierte Innovationen können sich als nicht vom Kunden gewünscht herauskristallisieren und ein komplettes Umdenken der Entscheider in den Verkehrsbetrieben erfordern. Angesichts des dynamischen Mobilitätsumfeldes könnten auch Experten nur unter Beachtung signifikanter Ungewissheiten langfristige Szenarien prognostizieren. Die Entwicklung der Haltestelle der Zukunft bedarf deshalb einer nutzerzentrierten Begleitforschung.

Die flächendeckende Einführung von autonom fahrenden Bussen wird nach Einschätzung der Fachexperten langfristig erfolgen. Diese Einführung hat große Auswirkungen auf den Verkehrssektor und die Haltestelle. Demnach kann der meist defizitäre Nahverkehr Kosteneinsparungen verzeichnen und sich als lukratives Marktsegment erweisen. Hierdurch können die Städte ihren Haushalt verbessern und durch einen kostengünstigen ÖPNV weitere Anreize für eine Verlagerung der Individualverkehre auf den ÖPNV bieten.

Autonom betriebene Drohnen und auch Seilbahnen sind weitere Mobilitätsmöglichkeiten, die langfristig in den Fokus rücken können. Der Klimawandel und dessen Folgen zeigen eine nachhaltigere Haltung der deutschen Bevölkerung im Umgang mit den Ressourcen der Erde. Diskussionen über die Einführung von CO2-Steuern bzw. Ausweitung von CO2-Zertifikaten zielen auf eine Reduzierung der Luftverkehre hin. Es ist davon auszugehen, dass eine Umsetzung dieser beiden Möglichkeiten eng an umweltschonende Techniken gebunden sein wird. Die Haltestelle wird entsprechend den technischen Möglichkeiten angepasst und neu definiert.

Die Ergebnisse der Befragung zeigen, dass die Haltestelle ein unentbehrliches Bindeglied zwischen der Bevölkerung und der Mobilität darstellt. Gemessen an dieser Erkenntnis ist die Haltestelle nach Auffassung der Autoren in der Fachliteratur unterrepräsentiert und bietet Potenzial für weitere Forschung. Die Fachexperten sehen in ländlichen Regionen einen verstärkten Bedarf an modernen MaaS-Konzepten.

Funding Open Access funding provided by Projekt DEAL.

Open Access Dieser Artikel wird unter der Creative Commons Namensnennung 4.0 International Lizenz veröffentlicht, welche die Nutzung, Vervielfältigung, Bearbeitung, Verbreitung und Wiedergabe in jeglichem Medium und Format erlaubt, sofern Sie den/die ursprünglichen Autor(en) und die Quelle ordnungsgemäß nennen, einen Link zur Creative Commons Lizenz beifügen und angeben, ob Änderungen vorgenommen wurden.

Die in diesem Artikel enthaltenen Bilder und sonstiges Drittmaterial unterliegen ebenfalls der genannten Creative Commons Lizenz, sofern sich aus der Abbildungslegende nichts anderes ergibt. Sofern das betreffende Material nicht unter der genannten Creative Commons Lizenz steht und die betreffende Handlung nicht nach gesetzlichen Vorschriften erlaubt ist, ist für die oben aufgeführten Weiterverwendungen des Materials die Einwilligung des jeweiligen Rechteinhabers einzuholen. 
Weitere Details zur Lizenz entnehmen Sie bitte der Lizenzinformation auf http://creativecommons.org/ licenses/by/4.0/deed.de.

\section{Literatur}

\section{Verwendete Literatur}

Arsenio E, Martens K, Di Ciommo F (2016) Sustainable urban mobility plans: bridging climate change and equity targets? Res Transp Econ 55:30-39

Braun V, Clarke V (2006) Using thematic analysis in psychology. Qual Res Psychol 3:77-101

Ceder AA, Butcher M, Wang L (2015) Optimization of bus stop placement for routes on uneven topography. Transp Res Part B Methodol 74:40-61

Chakour V, Eluru N (2016) Examining the influence of stop level infrastructure and built environment on bus ridership in Montreal. J Transp Geogr 51:205-217

Chen J, Liu Z, Zhu S et al (2015) Design of limited-stop bus service with capacity constraint and stochastic travel time. Transp Res Part E Logist Transp Rev 83:1-15

Choi W, Ranasinghe D, DeShazo JR et al (2018) Where to locate transit stops: cross-intersection profiles of ultrafine particles and implications for pedestrian exposure. Environ Pollut 233:235-245

Gu W, Gayah VV, Cassidy MJ et al (2014) On the impacts of bus stops near signalized intersections: models of car and bus delays. Transp Res Part B Methodol 68:123-140

Hensher DA (2017) Future bus transport contracts under a mobility as a service (MaaS) regime in the digital age: are they likely to change? Transp Res Part A Policy Pract 98:86-96

Hietanen S (2014) "Mobility as a Service"-The new transport model? Eurotransport 12(2):2-4

Ibeas Á, dell'Olio L, Alonso B et al (2010) Optimizing bus stop spacing in urban areas. Transp Res Part E Logist Transp Rev 46(3):446-458

Jittrapirom P, Caiati V, Feneri AM et al (2017) Mobility as a service: a critical review of definitions, assessments of schemes, and key challenges. Urban Plan 2(2):13-25. https://doi.org/10.17645/up. v2i2.931

Kamargianni M, Matyas M (2017) The business Eco-system of mobility as a service. In: 96th Transportation Research Board (TRB) Annual Meeting Washington DC, 8-12.01.. Bd. 2017

Kamargianni M, Li W, Matyas M et al (2016) A critical review of new mobility services for urban transport. Transp Res Proc 14:3294-3303

Kohli M (1978) „Offenes“ und „geschlossenes“ Interview: Neue Argumente zu einer alten Kontroverse. Soz Welt 29(1):1-25

Liggett R, Loukaitou-Sideris A, Iseki H (2001) Bus stop-environment connection: do characteristics of the built environment correlate with bus stop crime? Transp Res Rec 1760:20-27

Liu Z, Yan Y, Qu X et al (2013) Bus stop-skipping scheme with random travel time. Transp Res Part C Emerg Technol 35:46-56

Loukaitou-Sideris A (2006) Is it safe to walk? Neighborhood safety and security considerations and their effects on walking. J Plan Lit 20(3):219-232

Moeckel R, Nagel K (2016) Maintaining mobility in substantial urban growth futures. Transp Res Proc 19:70-80

Monzon A, Hernandez S, Cascajo R (2013) Quality of bus services performance: benefits of real time passenger information systems. Transp Telecommun 14(2):155-166

Newton RM, Thomas WH (1969) Design of school bus routes by computer. Socioecon Plann Sci 3(1):75-85

Rogge M, van der Hurk E, Larsen A et al (2018) Electric bus fleet size and mix problem with optimization of charging infrastructure. Appl Energy 211:282-295

Tavares AS, Gálvez C, de Albuquerque LWN et al (2015) Information on public transport: a comparison between information systems at bus stops. Proc Manuf 3:6353-6360

Tirachini A (2014) The economics and engineering of bus stops: spacing, design and congestion. Transp Res Part A Policy Pract 59:37-57

Tirachini A, Hensher DA, Rose JM (2014) Multimodal pricing and optimal design of urban public transport: the interplay between traffic congestion and bus crowding. Transp Res Part B Methodol 61:33-54 


\section{Weiterführende Literatur}

Kikuchi S, Vuchic VR (1982) Transit vehicle stopping regimes and spacings. Transp Sci 16(3):311-331 